\title{
Editorial: Knowledge Translation for Disease Control in Low- and Middle-Income Countries
}

\author{
Yodi Mahendradhata ${ }^{1 *}$ and Anna Kalbarczyk ${ }^{2}$ \\ 1 Department of Health Policy and Management, Faculty of Medicine, Public Health and Nursing, Universitas Gadjah Mada, \\ Yogyakarta, Indonesia, 2 Department of International Health, Johns Hopkins Bloomberg School of Public Health, Baltimore, \\ MD, United States
}

Keywords: knowledge translation, disease control, low- and middle-income countries, knowledge co-production, implementation research, citizen science

Editorial on the Research Topic

Knowledge Translation for Disease Control in Low- and Middle-Income Countries

Knowledge or awareness of evidence does not necessarily translate to policy and practice, as reported by Ezenwaka and Onwujekwe in this collection. The authors documented that many policy and decision-makers in their study are knowledgeable that evidence should inform policy decisions. Yet, only a few use evidence for developing policy. One important reason for the lack of commitment to the evidence-to-policy process is the policymaker's poor capacity to access, synthesize, and utilize evidence. Without sufficient capacity in knowledge translation (KT), evidence users will not have the capability of accessing and synthesizing information for evidence-informed decision making. The potential for shared learning will thus be misplaced.

We also know critical gaps in effective KT affect researchers and knowledge users globally. There remains a lack of knowledge about what $\mathrm{KT}$ is and how to do it, both in technical and soft skills (i.e. communication, networking, and relationship building). This influences a lack of institutional support, resulting in limited financial resources and few dedicated personnel supporting KT activities (1). Knowledge users (i.e. policymakers) have cited challenges accessing relevant, reliable, and locally relevant research (2). These barriers are well established and complex, particularly in low-and middle-income countries (LMICs).

Integrated KT (IKT) is a process that actively engages knowledge users in KT from the onset (i.e. research question prioritization and design) $(3,4)$. This co-production of research recognizes the need to engage knowledge users early, often in context-specific and creative ways. Strategies for engagement have historically separated the knowledge producers as experts and knowledge users as, just that, users of knowledge (5-7). However, these traditional approaches and silos are not working; they devalue the range of knowledge that different stakeholders can offer (i.e. indigenous tacit knowledge) and fail to account for structural issues that affect knowledge uptake (i.e. existing priorities and political will). This is why knowledge co-production through collaborative partnerships is much needed.

Niyibizi et al. suggest that citizen science promotes knowledge co-production through general public engagement in scientific research activities. Citizens actively contribute to science either with 
their intellectual effort or surrounding knowledge or with their tools and resources. It can inform local policymakers about perceptions and views and provide access to lay knowledge. This helps to formulate policies and design interventions relevant to the local context. Thus, it is rational and worthy to embed citizen science within the IKT in case there is a need to tailor knowledge for use within specific contexts and support the development of evidence-based decisions. A deliberate IKT approach that incorporates citizen science can be invaluable in improving the relevance and impact of research in the local community.

Mpando et al. highlight that IKT does not constitute a panacea but comes with its opportunities and challenges, requiring careful deliberation of whether the project circumstances and scope lend themselves to a systematic IKT approach. The importance of developing a stakeholder engagement strategy at the initiation of research activities cannot be overstated. However, organizational, political, and health system factors are constantly shifting, and therefore stakeholder engagement plans cannot remain static but instead need to embrace dynamism.

Training in implementation research (IR) holds great promise for building capacity to facilitate effective delivery of evidenceinformed interventions and enabling their scale-up. IR is growing globally with courses and training programs that cater to researchers and practitioners seeking to learn more about IR methodologies and approaches (8). However, Penkunas et al. report that current training approaches typically do not support the full range of learners who participate in IR teams (i.e. program implementers) and lack the content needed to become proficient in meaningfully presenting key IR results to policy audiences (9). IR involves more than one knowledge translation process. For these processes to work, multiple forms of expertise and experience must be mobilized at different phases of the research cycle and consider the level of structural, systemic, and strategic support available to decisionmakers. IR practitioners must develop the capacity to learn the specific dialects of policy and practice relevant to their initiatives.

The papers in this Research Topic also present novel approaches to engaging knowledge users for effective and responsive $\mathrm{KT}$, including videos. Videos can evoke powerful human emotions and explain anything involving many steps, as reported by Rahman et al. Some knowledge users have also expressed that videos are more desirable than text-based promotions or infographics (i.e. more traditional approaches to $\mathrm{KT}$ ). The 'show, not tell' way of storytelling through videos jumps across the complex nature of text-based knowledge sharing, and videos can help relevant stakeholders understand the "bigger picture" of public health interventions.

\section{REFERENCES}

1. Kalbarczyk A, Rodriguez DC, Mahendradhata Y, Sarker M, Seme A, Majumdar P, et al. Barriers and Facilitators to Knowledge Translation Activities Within Academic Institutions in Low- and Middle-Income Countries. Health Policy Plan (2021) 36(5):728-39. doi: 10.1093/heapol/ czaal 88

2. Edwards A, Zweigenthal V, Olivier J. Evidence Map of Knowledge Translation Strategies, Outcomes, Facilitators and Barriers in African
KT is a practical approach for improving health globally. By documenting, tailoring, and sharing what we know through research into policy and practice, we can continue to improve health globally. The papers included in this Research Topic collection have contributed to further understanding KT in LMICs. However, much still needs to be done to advance KT in LMICs, as derived from the papers in this Research Topic collection, including:

- A scoping review of the stakeholder landscape to inform IKT strategies (Mpando et al.).

- A more detailed understanding of IKT approaches and models linked to outcomes (Mpando et al.).

- Effectiveness trials of knowledge translation innovations (Rahman et al.).

- Further studies to provide insights on the impact or long term outcomes resulting from the implementation of citizen science embedded within an IKT approach (Niyibizi et al.).

- Build skills to strengthen the full potential of IR to be realized in LMICs, including the ability to work as part of a multidisciplinary team, engage stakeholders, and communicate research findings (Penkunas et al.).

- Invest in sustainable and continuous capacity-building activities to develop a critical mass of evidence users and set up institutional structure and processes to facilitate enhanced uptake of evidence into policy decisions (Ezenwaka and Onwujekwe).

The global COVID-19 pandemic has highlighted the importance of carefully communicating scientific data for use by policymakers (9). The response to the pandemic has been confronted by the proliferation of misinformation and poor access to evidence, which compound the ongoing challenges to knowledge translation in LMICs (10). The articles in this collection provide exciting paths forward, allowing us to critically assess past approaches and generate ideas for future engagement and research.

\section{AUTHOR CONTRIBUTIONS}

YM conceptualized the idea, developed the outline, developed the first draft, managed the revisions and finalized the manuscript. AK conceptualized the idea, developed the outline, developed the first draft and critically revised the manuscript. All authors checked and approved the final version submitted.

Health Systems. Health Res Policy Syst (2019) 17(1):16. doi: 10.1186/ s12961-019-0419-0

3. Plamondon KM, Pemberton J. Blending Integrated Knowledge Translation With Global Health Governance: An Approach for Advancing Action on a Wicked Problem. Health Res Policy Syst (2019) 17(1):24. doi: 10.1186/s12961-019-0424-3

4. Graham ID, McCutcheon C, Kothari A. Exploring the Frontiers of Research Co-Production: The Integrated Knowledge Translation Research Network Concept Papers. Health Res Policy Syst (2019) 17(1):88. doi: 10.1186/s12961019-0501-7 
5. Lawrence LM, Bishop A, Curran J. Integrated Knowledge Translation With Public Health Policy Makers: A Scoping Review. Healthc Policy (2019) 14 (3):55-77. doi: 10.12927/hcpol.2019.25792

6. Armstrong R, Waters E, Dobbins M, Anderson L, Moore L, Petticrew M, et al. Knowledge Translation Strategies to Improve the Use of Evidence in Public Health Decision Making in Local Government: Intervention Design and Implementation Plan. Implement Sci (2013) 8:121. doi: 10.1186/1748-5908-8-121

7. Graham ID, Kothari A, McCutcheon CIntegrated Knowledge Translation Research Network Project Leads. Moving Knowledge Into Action for More Effective Practice, Programmes and Policy: Protocol for a Research Programme on Integrated Knowledge Translation. Implement Sci (2018) 13 (1):22. doi: 10.1186/s13012-017-0700-y

8. Alonge O, Rao A, Kalbarczyk A, Maher D, Gonzalez Marulanda ER, Sarker M, et al. Developing a Framework of Core Competencies in Implementation Research for Low/Middle-Income Countries. BMJ Glob Health (2019) 4(5): e001747. doi: 10.1136/bmjgh-2019-001747

9. Mahendradhata Y, Kalbarczyk A. Prioritizing Knowledge Translation in Lowand Middle-Income Countries to Support Pandemic Response and Preparedness. Health Res Policy Syst (2021) 19(1):5. doi: 10.1186/s12961020-00670-1
10. El-Jardali F, Bou-Karroum L, Fadlallah R. Amplifying the Role of Knowledge Translation Platforms in the COVID-19 Pandemic Response. Health Res Policy Syst (2020) 18(1):58. doi: 10.1186/s12961-020-00576-y

Conflict of Interest: The authors declare that the research was conducted in the absence of any commercial or financial relationships that could be construed as a potential conflict of interest.

Publisher's Note: All claims expressed in this article are solely those of the authors and do not necessarily represent those of their affiliated organizations, or those of the publisher, the editors and the reviewers. Any product that may be evaluated in this article, or claim that may be made by its manufacturer, is not guaranteed or endorsed by the publisher.

Copyright (c) 2022 Mahendradhata and Kalbarczyk. This is an open-access article distributed under the terms of the Creative Commons Attribution License (CC BY). The use, distribution or reproduction in other forums is permitted, provided the original author(s) and the copyright owner(s) are credited and that the original publication in this journal is cited, in accordance with accepted academic practice. No use, distribution or reproduction is permitted which does not comply with these terms. 